\title{
Pharmacological evaluation of novel PAC1 receptor antagonist on oxaliplatin- induced cold allodynia in mice
}

\author{
Takahiro Ueda', Ryota Nagashima ${ }^{1}$, Ai Watanabe ${ }^{1}$, Takuya Okada $^{1}$, Naoki Toyooka ${ }^{1}$, \\ Atsuro Miyata ${ }^{2}$, Takashi Kurihara ${ }^{2}$, Ichiro Takasaki ${ }^{1}$ \\ ${ }^{I}$ Grad. Sch. Sci. \& Eng. Res., Univ. Toyama, Japan, ${ }^{2}$ Grad. Sch. Med. \& Dent. Sci., Univ. Kagoshima, Japan
}

Oxaliplatin (OXA) is a platinum-based anticancer agent widely used for metastatic colorectal cancer. However it is efficacious against tumors, it induce severe peripheral neuropathy such as numbness and cold allodynia of the hands and feet. To attenuate these side effects, several drugs are used, however there are few analgesics to control the pain.

Pituitary adenylate cyclase-activating polypeptide (PACAP) is a pleiotropic neuropeptide, which functions as a neurotransmitter and neuromodulator. Recently, we found that intrathecal (i.t.) injection of PACAP induces long-term cold allodynia in mice, suggesting that PACAP-PACAP receptor systems in the spinal cord are involved in cold allodynia and that PACAP receptor antagonist could be a novel analgesics. More recently, we have developed a novel and smallmolecule antagonist of PAC1 receptor (named PA-8) by in silico screening and in vitro/vivo pharmacological assays. Furthermore, we synthesized derivative compounds of PA-8 named PA-810 and PA-81004. In this study, we investigated the involvement of PACAP and its receptor in OXA-induced cold allodynia and examined the effects of PA-8, PA-810 and PA-81004 on OXA-induced cold allodynia

Cold allodynia of the hind paw was assessed using the acetone test. Briefly acetone drop (10 \&\#181;L) applied to the planter skin, and the time spent in elevating and licking the stimulated hind paw was measured for $60 \mathrm{~s}$.

Single administration of OXA ( $2 \mathrm{mg} / \mathrm{kg}$, i.p.) significantly increased the time of elevating and licking of the hind paw in response to acetone stimulation, from 1 to 10 days after the administration, compared to the vehicle administration group. We used mice on 4 days after OXA administration. I.t. injection of PACAP 6-38 (peptide antagonist of PACAP receptor, $0.1 \mathrm{nmol})$ and PA-8 (0.1-10 nmol) ameliorated the OXA-induced cold allodynia, suggesting that spinal PAC1 receptor is involved in the OXA-induced cold allodynia. Next, we examined the effects of systemic administration of PA-8, PA-810 and PA-81004 on OXA-induced cold allodynia. As a result, both intraperitoned and oral administration $(3-30 \mathrm{mg} / \mathrm{kg})$ dose-dependently alleviated the OXA-induced cold allodynia. The present results suggest that PA-8, PA-810 and PA -81004 may become oral active analgesics against chemotherapy-induced peripheral neuropathy. 